\title{
Estudiantes Universitarios: prosumidores de recursos digitales y mediáticos en la era de internet
}

\author{
Mónica Bonilla-del-Río $\left({ }^{*}\right)$, José Manuel Diego-Mantecón $\left(^{*}\right)$ y Francisco Javier Lena-Acebo $\left(^{*}\right)$ \\ $\left.{ }^{*}\right)$ Universidad de Cantabria - España
}

\section{RESUMEN}

A pesar de la relevancia que los recursos digitales y mediáticos han adquirido en nuestra sociedad, la adquisición de competencias para su uso adecuado y responsable en el contexto educativo no está garantizada. Este trabajo busca analizar los hábitos de producción y utilización de los recursos digitales y mediáticos de una muestra de 252 universitarios españoles, con el objetivo de conocer su competencia digital y su aprovechamiento para un uso educativo. Los resultados revelan una competencia media/alta de los universitarios en cuanto al manejo de los recursos mediáticos y digitales, así como una percepción positiva de la red como fuente valiosa de la que adquirir conocimiento y ampliar información. Admiten utilizar los recursos digitales y mediáticos para trabajar de forma colaborativa y ayudarse en tareas académicas, aunque no producen contenido académico o información en plataformas educativas; es decir, se consideran consumidores mediáticos, pero no productores. Se observó además que los universitarios mayores de 25 años son prosumidores más activos, en lo que se refiera a contenidos e información relacionada con temas académicos, que los estudiantes menores de 26 años, quienes utilizan más las redes para producir y compartir datos personales.

Palabras Clave: Alfabetización mediática, competencia mediática, competencia digital, internet, nativos digitales.

\section{University students: Prosumers of digital and media resources in the internet era}

\section{ABSTRACT}

Despite the importance that digital and media resources have reached in our society, the acquisition of competencies for their suitable and responsible use in the educational context is not guaranteed. This study seeks to analyze the habits of production and use of digital and media resources of a sample of 252 Spanish university students, aiming to know their digital competence for an educational purpose. The results reveal a medium/high competence of the university students regarding the management of digital and media resources, as well as a positive perception of the network as a valuable source from which to acquire knowledge and expand information. They admit using digital and media resources to work collaboratively and help each other in academic tasks, although they do not produce academic content or information on educational platforms; that is, they considered themselves media consumers, but not producers. It was also observed that university students over the age of 25 are more active prosumers - in terms of academic content and/or information - than students under 26, who use the networks more to produce and share personal data.

Keywords: Media Literacy, media competence, digital competence, internet, digital natives.

\section{Introducción}

En la actualidad los procesos comunicativos se producen en un entorno multipantalla donde los recursos digitales y mediáticos adquieren gran relevancia a nivel profesional, social, económico, político o de entretenimiento, por lo que las instituciones educativas no han de permanecer ajenas a este contexto mediático (De Casas-Moreno, Caldeiro, y Romero-Rodríguez, 2018; Go- zálvez, García-Ruiz, y Aguaded, 2014). Las posibilidades de participación que ofrecen estos medios y dispositivos convierten a los usuarios en prosumidores ${ }^{1}$, pasando de ser meros receptores de la información a tener la posibilidad de producir y compartir sus propios contenidos (González-Fernández, Ramírez-García, y Salcines-Talledo, 2017). El hecho de crecer en un contexto mediático rodeado de avances tecnológicos no garantiza que se haga un uso adecuado y responsable de estos recursos (Fajardo, Vi-

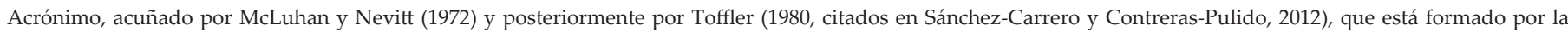
fusión original de las palabras en inglés producer (productor) y consumer (consumidor)
} 
llalta y Salmerón, 2016). Diversos estudios ponen de manifiesto que la simple utilización de los recursos digitales y mediáticos por parte de las nuevas generaciones no implica necesariamente su aprovechamiento, ni la consecución de un nivel alto de competencia digital (Acosta-Silva, 2017; Carrasco, Sánchez, y Carro, 2015; Cabra y Marciales, 2009). Estos estudios cuestionan el planteamiento de Prensky (2001) sobre los nativos digitales, que otorga al individuo la adquisición de la competencia digital por el simple hecho de haber nacido en la era tecnológica.

En este sentido, diversos organismos a nivel internacional, como la Comisión Europea y la Organización de las Naciones Unidas para la Educación, la Ciencia y la Cultura (UNESCO por sus siglas en inglés) reconocen la importancia de que las instituciones educativas ofrezcan a la ciudadanía una alfabetización acorde a las necesidades y características de la sociedad actual. En particular, advierten de la importancia de promover el desarrollo de la "alfabetización mediática" con el fin de favorecer respuestas educativas que contemplen el uso informado y crítico de los medios de comunicación, para mejorar la participación social y los procesos de comunicación en diversos contextos. La alfabetización mediática es la capacidad de acceder y hacer uso de los medios de comunicación de forma adecuada y responsable (Comisión Europea, 2009), evaluando la información de manera crítica, así como las técnicas empleadas por los propios medios, y sus efectos, para comunicar en diferentes contextos (UNESCO, 2008).

Se pone de manifiesto, por tanto, la necesidad de promover una educación especializada que contribuya al uso y consumo adecuado de los recursos digitales y mediáticos, y que permita la prevención y detección de riesgos o comportamientos inadecuados (Rotta, 2015). De acuerdo con Benítez, Manrique y De Mier (2014) esta educación ha de ser multidimensional valorando las diversas dimensiones humanas, socioeconómicas, políticas y pedagógicas, y desarrollando la "competencia mediática" y "digital" en todos los ámbitos y etapas educativas (Caldeiro y Aguaded, 2015; López y Aguaded, 2015). La competencia mediática hace referencia a la capacidad de percepción, análisis y disfrute de los mensajes y estímulos (ej. sonidos o imágenes) presentes en los medios de comunicación, a través de una utilización crítica que permita la satisfacción de las necesidades informativas, comunicativas y formativas generadas en la cotidianeidad del entorno (Comisión Europea, 2011). Es necesario, por tanto, superar toda visión reduccionista en torno al simple manejo instrumental de los recursos digitales y mediáticos, y avanzar hacia perspectivas que faciliten su utilización de forma inteligente, así como impulsar el desarrollo de habilidades y actitudes que favorezcan el espíritu crítico y reflexivo, el compromiso cívico y cultural, y la actividad creativa (Buckingham, 2008; Ferrés y Piscitelli, 2012; Gozálvez y Aguaded, 2012).

La competencia digital, impulsada inicialmente por la Unión Europea (European Council, 2006), es una competencia clave para alcanzar un desarrollo personal, social y profesional ajustado a las demandas del mundo globalizado. Esta competencia, recogida también en la actual Ley Orgánica para la Mejora de la Calidad Educativa (LOMCE) en España, se define como el conjunto de conocimientos, habilidades y actitudes necesarias para adecuarse a los cambios producidos por las tecnologías y ser competente en el entorno digital y la comunicación (Ministerio de Educación, Cultura y Deporte, 2015). De acuerdo con Pérez-Rodríguez y Delgado-Ponce (2012) la competencia mediática y la competencia digital han de ser complementarias con el fin de conseguir una ciudadanía dotada de los conocimientos, actitudes y destrezas necesarias para desenvolverse de manera satisfactoria en los entornos virtuales y mediatizados, así como para hacer un uso efectivo de las nuevas Tecnologías de Infor- mación y Comunicación (TIC). En esta línea, Gutiérrez y Tyner (2012) señalan los riesgos de limitar la educación mediática al desarrollo de la competencia digital y de reducir esta última a su dimensión instrumental; es decir, limitarla a conocimientos técnicos y al manejo de dispositivos, trasladando a un segundo plano los valores y las actitudes necesarias para realizar una utilización eficaz de los recursos digitales y mediáticos.

\section{Apropiación de los recursos digitales y mediáticos por parte de la ciudadanía: retos y posibilidades}

Los medios de comunicación y los dispositivos tecnológicos influyen de manera significativa en la formación cultural de la población, especialmente en los niños y jóvenes (Sesento, 2015). Las nuevas generaciones se desarrollan en un entorno en el que los recursos digitales y mediáticos forman parte de su cotidianeidad, lo que influye en sus preferencias, hábitos y relaciones interpersonales (Scolari, 2016). El acceso a internet, tal y como plantean Ortega, González, y Pérez (2015), se produce cada vez a edades más tempranas y la interacción y posesión de dispositivos les convierten en protagonistas de los contenidos que consumen, crean y comparten a través de entornos virtuales (García-Galera y Valdivia, 2014). De hecho, las actividades de ocio, los procesos de comunicación, el acceso a la información y el aprendizaje de los jóvenes tienen una relación directa con los recursos digitales y mediáticos, tanto dentro como fuera del ámbito educativo. Sánchez-Carrero y Contreras-Pulido (2012) hacen referencia al prosumidor mediáti$\mathrm{CO}$ - ciudadano con capacidad para producir y consumir información, así como contenidos digitales y audiovisuales, y que emplea un amplio abanico de herramientas multimedia y estrategias que posibilitan procesos de consumo y creación de información en los diferentes entornos virtuales. Este ciudadano tiene además capacidad para valorar y analizar los contenidos de manera autónoma; es decir posee autonomía mediática, tal y como señalan $\underline{\text { Aguaded, }}$ Caldeiro, y Rodríguez (2015).

A pesar de que a las nuevas generaciones se les atribuye habitualmente la etiqueta de prosumidores mediáticos, estudios como el de Kennedy et al. (2007) revelan que la producción e intercambio de contenidos propios, a través del uso de los recursos digitales y mediáticos, es escaso entre los más jóvenes. Sarsa (2014) sugiere que esta escasa participación de los jóvenes a la hora de adquirir el rol de productores/consumidores mediáticos podría deberse a una falta de interés y de conocimiento en el manejo de diferentes plataformas. Surge la necesidad, por tanto, de fomentar estrategias educativas que permitan al estudiante adquirir habilidades y actitudes que posibiliten su evolución desde un enfoque meramente consumidor a uno mixto, combinando los contextos de expresión/recepción y favoreciendo la alfabetización mediática mediante la formación de emisores y receptores reflexivos (García-Ruiz, Ramírez, y Rodríguez, 2014).

En particular, Ricoy y Da Silva (2016) señalan que para que los jóvenes universitarios puedan hacer frente, con ciertas garantías, a los retos de la sociedad y obtener un aprovechamiento óptimo de los dispositivos es necesario que se les forme en el desarrollo de habilidades que favorezcan: la búsqueda de información contrastada, la resolución de problemas, la comunicación eficaz en entornos virtuales, y la autonomía para crear y compartir contenidos. Estos aprendizajes estructurados de los individuos en formación adquieren mayor relevancia y significatividad cuando la educación formal, en cualquiera de sus etapas educativas, está vinculada con la vida fuera del ámbito académico, por lo que la instrucción ha de involucrar entornos de aprendizaje reales y virtuales, así como entornos colaborativos (Tyner, Gutiérrez, y Torrego, 2015). 


\subsection{La responsabilidad de las instituciones educativas para im-} pulsar la alfabetización mediática

El reto educativo en términos de alfabetización mediática se establece en la necesidad de formar a ciudadanos libres, participativos, con capacidad crítica y con un nivel de compromiso social y autonomía que les permita un consumo y una producción responsable y satisfactoria a través de los recursos digitales y mediáticos (Monreal, Parejo, y De la O, 2017). Investigaciones recientes sobre el diagnóstico de la competencia digital y mediática de los jóvenes sugieren que es necesario continuar promoviendo la formación de las nuevas generaciones, con el fin de mejorar su nivel competencial. Las instituciones educativas no han de permanecer, por tanto, ajenas al reto de adecuar los procesos educativos a esta realidad (Iglesias y González, 2012; Grijalva y Moreno, 2016; Grijalva y Urrea, 2017; Liesa, Vázquez, y Lloret, 2016; Ferrés, 2017). Del Valle-Rojas, Denegri-Coria, y Chávez-Herting (2012) revelan que los estudiantes carecen de las habilidades y competencias necesarias para discernir entre la ingente cantidad de información disponible aquella que es valiosa, no siendo tampoco capaces de identificar las diferentes técnicas de persuasión de los medios en cuanto al manejo de la información.

A nivel internacional ya se han planteado diferentes modelos para desarrollar la alfabetización mediática en el contexto universitario, específicamente en países desarrollados (Valverde, Reyes, y Espinosa, 2012). Algunas de estas experiencias llevadas a cabo en las universidades han mostrado que los talleres sobre alfabetización mediática son muy útiles, constatándose la mejora del alumnado en habilidades de comprensión y organización de la información, así como en el desarrollo de su capacidad crítica y comunicativa (Izquierdo, Renés, y Gómez, 2013). Cabero y Guerra (2011) destacan que este tipo de talleres favorece también destrezas relacionadas con la búsqueda, análisis y evaluación de la información; la resolución de problemas y la competencia tecnológica; la utilización efectiva de herramientas de producción o creación; así como la comunicación y la colaboración con otros usuarios. Por último, Soep (2012) resalta que la creación de contenidos mediáticos y digitales por parte de los jóvenes, mediante la experimentación, les permite desarrollar dimensiones clave en la alfabetización mediática como el aprendizaje por descubrimiento, el análisis y el proceso de movilización en red. En particular, la programación de plataformas interactivas online les proporciona la posibilidad de crear, transformar y compartir con otros usuarios sus propios contenidos.

Teniendo en cuenta la revisión bibliográfica anterior, el presente estudio tiene como objetivo principal analizar la utilización que hacen los universitarios de las redes sociales e internet, con el fin de conocer sus hábitos como prosumidores mediáticos y el uso que hacen de los recursos digitales para mejorar su aprendizaje. Como hemos señalado anteriormente, el conocimiento, necesidades e intereses del alumnado en lo referente al uso de los recursos digitales y mediáticos puede contribuir a un entendimiento y aprovechamiento más responsable y efectivo de los medios y dispositivos, tanto en su uso cotidiano como en el ámbito académico. Todo ello, conducirá a formar ciudadanos más competentes digitalmente que se adapten a las demandas de la actual sociedad de la información y el conocimiento.

\section{Método de investigación}

La investigación se establece en torno a tres dimensiones principales con el propósito de obtener información precisa sobre el objeto de estudio: (1) Prosumidor mediático, (2) Competencia digital, (3) Relevancia de las redes sociales e internet. En lo referente a la dimensión prosumidor mediático se abordan aspectos relacionados con los hábitos del estudiante universitario sobre el uso de las redes sociales e internet tales como: la producción y consumo de contenidos personales (ej. edición de fotos o videos) que se comparten en la red con un fin meramente de esparcimiento; la producción y consumo de contenidos académicos, o información, que se difunden en la red con el objetivo de trasmitir conocimiento; o la utilización de la redes para trabajar y aprender de forma colaborativa. La dimensión competencia digital atiende a aspectos relacionados con las destrezas, actitudes y conocimiento de los jóvenes universitarios en cuanto al manejo de los recursos digitales y mediáticos, la resolución de conflictos relacionados con la utilización de estos recursos; y la tendencia a mostrar posturas respetuosas y valores cívicos en entornos virtuales. Por último, la dimensión relevancia de las redes sociales e internet busca recabar información acerca de la percepción que tienen los universitarios sobre la importancia e influencia de internet, así como de la repercusión de este medio en su vida diaria y en la de los demás.

\section{Proceso de investigación}

De acuerdo con los objetivos de la investigación se optó por emplear un enfoque cuantitativo de análisis de datos, que a través de la codificación de la información permita identificar patrones y hábitos de los sujetos a evaluar. Como estrategia de investigación se utilizó la encuesta, ya que permite obtener y elaborar datos de modo eficaz en un periodo de tiempo relativamente corto (Anguita et al., 2003). Como método de recogida de datos se utilizó el cuestionario. Este se diseñó en formato digital para ser utilizado online, y contiene dos partes. La primera parte recaba datos personales de los sujetos como la edad, el sexo, la universidad de pertenencia, la carrera que cursan, y las redes sociales que frecuentan. La segunda parte está compuesta por una batería de 30 ítems presentados en una escala de cuatro puntos, donde los puntos uno (Nunca) y dos (Rara vez) representan valores negativos y los puntos tres (A menudo) y cuatro (Siempre) valores positivos. De acuerdo con Diego-Mantecón (2013) se empleó una escala "par" para evitar una respuesta neutra que pudiera reducir la calidad de los datos recabados. En esta escala de cuatro puntos una media aritmética de 2,5 representa la neutralidad. Los 30 ítems se diseñaron en relación a las tres dimensiones a evaluar: (1) Prosumidor mediático, (2) Competencia digital, (3) Relevancia de las redes sociales e internet. Se diseñaron diez ítems aproximadamente por cada dimensión y se combinaron estratégicamente a la hora de ser presentados, para no guiar las respuestas de los sujetos participantes.

Previo a la administración del cuestionario, los ítems fueron revisados para incrementar su validez y consistencia interna (Geller, Vinokurov, y Martin, 2005). Para aumentar su validez interna, y verificar que evaluaban los aspectos pretendidos por los investigadores, éstos fueron revisados por un comité de expertos en el campo de las TIC. Para mejorar su consistencia interna - es decir, asegurar la comprensión de los ítems por todos los sujetos de la muestra - los ítems se administraron a un grupo de 22 universitarios de perfil similar a los de la muestra principal. Durante estos dos procesos se eliminaron o reformularon aquellos ítems que conducían a respuestas sesgadas o resultaban confusos.

Se optó por una estrategia de conveniencia para la selección de la muestra, que asegurase la recogida de datos de un número aceptable de sujetos. Se invitó por lo tanto a participar, vía correo electrónico, a estudiantes del Grado en Educación Infantil y del Máster en Formación del Profesorado de Educación Secundaria pertenecientes a tres universidades públicas españolas, a las que los investigadores tenían acceso. Se obtuvo respuesta del 93\% de los sujetos que recibieron la invitación, generándose una mues- 
tra total de 252 participantes (219 estudiantes de Grado y 33 de Máster). Los estudiantes encuestados (203 mujeres y 49 hombres) tienen edades que comprenden entre los 18 y 38 años. En particular $118(46,8 \%)$ de ellos están en el rango de 18 a 25 años y 134 $(53,2 \%)$ en el rango de 26 a 38 años. Conviene destacar que la diferencia de representatividad de las mujeres frente a los hombres se debe a que los estudiantes de los Grados en Infantil son mayoritariamente mujeres. Todos los sujetos de la muestra estaban familiarizados con las redes sociales e internet. El 96,6\% de ellos declaró ser usuarios de WhatsApp, el 95,9\% de YouTube, un 85\% de Facebook, el 83\% de Instagram, el 45\% de Twitter, y un 22,4\% de Snapchat. Otras redes como Musically, Pinterest o LinkedIn fueron reportadas por menos del 3\% de los encuestados.

\section{Análisis de datos y resultados}

Para evaluar la consistencia del instrumento se aplicó un análisis factorial con rotación ortogonal. Antes de computar el análisis factorial se hizo un escrutinio de los datos para valorar su grado de adecuación a la muestra. Se computó una matriz de correlaciones para eliminar aquellos ítems que mostraban una baja correlación (con valores inferiores a 0.20) y que, por lo tanto, no son recomendables para aplicar análisis factorial. También se computó el test de adecuación muestral de Kaiser Meyer-Olkin (KMO) y la prueba de esfericidad de Bartlett arrojando un valor de 0.729 con un $p<0.00005$, lo que sugiere que los datos son adecuados para aplicar análisis factorial.

Para extraer un número óptimo de factores se consideró el criterio de Kaiser y el test de Cattel. El criterio de Kaiser sugiere extraer el número de factores correspondientes a los valores propios mayores que uno, y el test de Cattel aquellos que indica el punto de inflexión del gráfico de sedimentación (Pallant y Manual, 2010). En este caso, se optó por la extracción de los siete factores recomendados por el criterio de Kaiser, ya que permitió una interpretación más completa de los datos. Los siete factores extraídos explican una varianza alta del 63.9\%. En la Tabla 1 se muestran los factores extraídos con sus ítems, y sus respectivas cargas factoriales.

Tabla 1. Factores extraídos y las cargas de cada uno de los ítems

Factores Ítems

Productor y

difusor de

contenidos

personales

Usuario colaborativo

Usuario

Responsable

Comparto fotos mías en la red

Hago fotos de donde estoy para compartirlas

Me hago selfies y los comparto en internet

Creo vídeos en los que salgo y los comparto

Nos ayudamos con los trabajos y exámenes por WhatsApp u otro chat

Uso grupos de chat para enterarme de los trabajos que hay que hacer para clase

Me comunico por internet para hacer trabajos colaborativos con mis compañeros

Tengo un comportamiento adecuado y respetuoso en las redes

Utilizo los mismos valores de respeto en internet que en la vida real

Conozco los riesgos de compartir información privada por internet

Los contenidos digitales que comparto pueden influir en la forma de pensar de otros

Crear vídeos y compartirlos ayuda a ser popular

medio

Influyente

Los contenidos de internet influyen en mi manera de pensar y actuar

Amplío lo que me enseñan en clase con información de internet

Consumidor mediático

Busco estar actualizado

Busco información en vídeos para comprender mejor algunos temas

Soy capaz de resolver un problema informático con mi dispositivo móvil u ordenador

Competente digital

Productor y

difusor

contenidos

mediáticos
, 264

\section{Cargas factoriales}

1

, 823

, 815

, 810

, 740

, 867

, 838

,680

, 835

,791

, 713

\section{, 726}

, 596 
El Factor 1 hace alusión a un Usuario productor y difusor de contenidos personales, al incluir ítems como 'Me hago selfies y los comparto en internet', 'Creo vídeos en los que salgo y los comparto' o 'Comparto fotos mías en la red'. El Factor 2 se corresponde con un Usuario colaborativo, que utiliza las redes para trabajar e interaccionar con sus compañeros, al contener los siguientes ítems: ‘Nos ayudamos con los trabajos y exámenes por WhatsApp u otro chat', 'Me comunico por internet para hacer trabajos colaborativos con mis compañeros' y 'Uso grupos de chat para enterarme de los trabajos que hay que hacer para clase'. El Factor 3 apunta hacia un Usuario responsable en los medios y las redes, al localizarse en este factor ítems como 'Tengo un comportamiento adecuado y respetuoso en las Redes' o 'Utilizo los mismos valores de respeto en internet que en la vida real'. El Factor 4 hace referencia a Internet como medio influyente en cuanto a los contenidos que los usuarios comparten (ej. 'Los contenidos digitales que comparto pueden influir en la forma de pensar de otros' y 'Crear vídeos y compartirlos ayuda a ser popular') y los que encuentran en la red (ej. 'Los contenidos de internet influyen en mi manera de pensar y actuar'). El Factor 5 se identifica con un Consumidor mediático que se nutre principalmente de contenidos e información académica. Así lo evidencian los ítems 'Amplío lo que me enseñan en clase con información de internet', 'Busco estar actualizado' o 'Busco información en vídeos para comprender mejor algunos temas'. El Factor 6 se corresponde con un Usuario competente digitalmente al incluir afirmaciones como: 'Soy capaz de resolver un problema informático con mi dispositivo móvil u ordenador' o 'Prefiero hacer trabajos en digital que en papel'. Los ítems que convergen en el Factor 7 - 'Edito información en Wikipedia sobre temas que conozco' y 'Comparto mis propios contenidos públicamente para que otros aprendan' - apuntan hacia un Usuario productor y difusor de contenidos mediáticos (no relativos al ámbito personal), que tiene el objetivo de transmitir conocimiento para que otros aprendan a través de las redes.

Una vez extraídos y etiquetados los factores, y tras asegurar su uni-dimensionalidad, se computó la alfa de Cronbach para conocer su fiabilidad (Wells y Wollack, 2003). Como muestra la Tabla 1, tres factores mostraron una consistencia interna aceptable con alfas entre 0,71 y 0,84 , y cuatro mostraron una consistencia media-baja con valores entre 0,5 y 0,61 . La consistencia media baja de algunos de los factores se debió, en gran parte, al reducido número de ítems por factor. Por ejemplo, el Factor 7 está constituido únicamente por dos ítems. En general los ítems mostraron cargas factoriales altas: nueve de ellos mostraron valores superiores a 0,80 , ocho arrojaron valores superiores a 0,70 , y únicamente cuatro mostraron valores por debajo de 0,60 . Esto sugiere que los factores extraídos muestran una consistencia aceptable y que su fiabilidad puede aumentar refinando aquellos ítems con valores por debajo de 0,70 e incluyendo, en los factores con dos o tres ítems, algún otro elemento que permita definir mejor el constructo que representan.

Tras la extracción de los factores, y una vez realizados los test de fiabilidad, se procedió a realizar el análisis de los mismos calculando la puntuación media de cada factor.

Tabla 2. Puntuación media por factor

\begin{tabular}{llcc}
\multicolumn{1}{c}{ Factores } & Medias & Desviación Típica \\
1. & Productor y difusor de contenidos personales & 2,25 & 0,68 \\
2. Usuario colaborativo, utiliza las redes para trabajar con otros & 3,01 & 0,73 \\
3. Usuario responsable & 3,60 & 0,50 \\
4. Internet como medio influyente & 2,29 & 0,62 \\
5. Consumidor mediático, se nutre de contenidos e información académica & 2,90 & 0,48 \\
6. Usuario competente digitalmente & 3,00 & 0,30 \\
7. Productor y difusor de contenidos mediáticos (no contenidos personales) & 1,51 & 0,57
\end{tabular}

Las puntuaciones medias indicaron que los estudiantes universitarios encuestados rara vez $(M=2,25)$ utilizan internet para compartir datos personales, pero a menudo sí lo utilizan como medio para trabajar de forma colaborativa $(M=3,01)$ y ayudarse en las tareas académicas. Por otro lado, señalan que rara vez editan en plataformas como Wikipedia o comparten sus contenidos públicamente para que otros aprendan $(\mathrm{M}=1,51)$. No consideran que los contenidos que ellos comparten puedan tener un impacto en otros $(M=2,29)$, aunque si tienden a creer que, a menudo, internet es una fuente de la que poder nutrirse y ampliar información $(\mathrm{M}=2,90)$. Los encuestados consideran que son, a menudo
$(\mathrm{M}=3,00)$, competentes en el manejo de dispositivos informáticos e internet, y que se comportan de manera responsable y educada en las redes $(M=3,60)$.

Para obtener información específica respecto a la variable edad, se computó el test Mann-Whitney U, en cada uno de los factores, ya que de acuerdo con $\underline{\operatorname{Nachar}(2008)}$ es uno de los test más útiles para detectar diferencias significativas entre grupos, cuando se trabaja con datos no paramétricos. Como muestra la Tabla 3, los análisis revelaron diferencias significativas en cuanto a la variable edad en cuatro de los siete factores.

Tabla 3: Puntuaciones medias de los factores en relación a la edad

Factores

1. Productor y difusor de contenidos personales
Menores de 26

Media

2,46
Mayores de 25

Media

2,08
DT

0,65
Mann-Whitney U Test

$\begin{array}{cc}\mathrm{U} & \mathrm{p} \\ 5523,00 & \mathbf{0 , 0 0 0}\end{array}$




\section{Factores}

2. Usuario colaborativo, utiliza las redes para trabajar con otros

3. Usuario responsable

4. Internet como medio influyente

5. Consumidor mediático, se nutre de contenidos académicos

6. Usuario competente digitalmente

7. Productor y difusor de contenidos mediáticos (no personales)
Menores de 26

3,20

3,53

2,30

2,79

2,88

1,43

0,50
0,50
0,611
0,41
0,44
0,51

Mayores de 25

\begin{abstract}
2,85
\end{abstract}
3,64

2,29

2,98

2,95

1,59
0,82
0,48
0,63
0,50
0,54
0,60

6135,50

6750,00

7731,50

6028,00

7241,00

6796,50
0,002

0,055

0,759

0,001

0,239

0,042

En cuanto al Factor 1 -Usuario productor y difusor de contenidos personales - se observó que los más jóvenes (de edades comprendidas entre los 18 y 25 años) crean y comparten significativamente más sus contenidos personales en las redes $(M=2,46, n=118)$ que sus compañeros mayores de 25 años $(\mathrm{M}=2,08, \mathrm{n}=134), U=5523, \mathrm{Z}=-4,15, P=0.000$. Los más jóvenes usan también significativamente más las redes para trabajar de forma colaborativa $(\mathrm{M}=3,20)$ que los mayores $(\mathrm{M}=2,85), U$ $=6135, Z=-3,11, P=0.002$. Por el contrario, los mayores de 25 años parecen utilizar significativamente más $(P=0.001)$ internet para ampliar información sobre lo que aprenden en el aula, y para estar más actualizados $(\mathrm{M}=2,98)$, que los más jóvenes $(M=2,79), U=6028, Z=-3,34$. De la misma manera, los mayores editan en Plataformas Web, o comparten sus contenidos públicamente para que otros aprendan, de manera más significativa $(\mathrm{M}=1,59, P=0.002)$ que sus compañeros más jóvenes $(\mathrm{M}=1,43)$, $U=6796, Z=, 042$. No se encontraron diferencias significativas en cuanto a su comportamiento en las redes (Factor 3), ni sobre su percepción de internet como un medio influyente (Factor $4)$, ni tampoco respecto a su competencia en el manejo digital (Factor 6).

\section{Discusión y conclusiones}

Como hemos argumentado a lo largo de este artículo, los recursos digitales y mediáticos han adquirido una relevancia fundamental en el contexto actual y se han establecido como elementos esenciales de la sociedad, ya no solo como facilitadores de la comunicación y de las relaciones interpersonales sino, también como factores clave en la formación del individuo. El objetivo principal de este estudio consistió en conocer la utilización que los universitarios españoles hacen de las redes sociales e internet, investigando sus hábitos como prosumidores mediáticos, su competencia digital, y su percepción sobre la influencia de este medio en la vida diaria de cada uno.

Los resultados revelan que los estudiantes universitarios encuestados no utilizan con asiduidad las redes sociales e internet como un medio de entretenimiento en el que crear y compartir contenidos personales, pero sí las emplean a menudo para trabajar de forma colaborativa y ayudarse en tareas académicas. Aunque no producen y comparten contenido académico o información en plataformas como Wikipedia para que otros aprendan, sí consideran internet como una fuente valiosa de la que poder adquirir conocimiento y ampliar información - es decir, no se consideran productores, pero sí consumidores mediáticos. Estos resultados coinciden con los obtenidos por De Oliveira (2016) y ponen de manifiesto el valor de los espacios virtuales como herramienta educativa y de apoyo en el desarrollo de prácticas formativas, para la construcción de conocimiento y la cooperación entre estudiantes.

Los estudiantes encuestados se consideran competentes en el manejo de dispositivos informáticos e internet, y destacan un comportamiento responsable y adecuado en las redes, resultados que concuerdan con los obtenidos por Castellanos, Sánchez, y Calderero (2017). Aunque consideran internet como una fuente de información sobre la que poder nutrirse, no creen que los contenidos que se comparten puedan influenciar a otros o cambiar su manera de pensar.

Es importante resaltar que se encontraron diferencias significativas respecto a la variable edad en varios de los factores. Los estudiantes más jóvenes (menores de 25 años) producen y comparten en las redes significativamente más contenidos personales (como entretenimiento) que los mayores de 25 años. Por el contrario, los universitarios de mayor edad producen contenido académico, o información, en plataformas como Wikipedia y a su vez consumen estos contenidos significativamente más que los de menor edad. Los universitarios de mayor edad son por lo tanto prosumidores más activos, que los más jóvenes, en lo que se refiera a contenidos e información relacionada con temas académicos. A diferencia de los mayores, los jóvenes tienden a utilizar significativamente más las redes para trabajar de forma colaborativa. No se encontraron diferencias significativas en cuanto a comportamiento responsable, ni respecto a la percepción de internet como un medio influyente. Tampoco se observaron diferencias en relación a su competencia digital.

Los resultados anteriores evidencian que el estudiante, con la edad, va adquiriendo más consciencia sobre la utilidad de los recursos mediáticos y digitales para fines académicos, dejando de utilizarlos como meros instrumentos de entretenimiento o socialización para comenzar a utilizarlos como herramientas educativas. En este sentido la impartición de talleres centrados en explotar las posibilidades de estos recursos en el contexto académico podría resultar enriquecedor, especialmente para los más jóvenes que, aunque admiten una competencia digital aceptable no se sienten tan seguros a la hora de utilizar estos recursos mediáticos y digitales como herramienta educativa.

\section{Apoyos}

Este trabajo se ha elaborado en el marco de Alfamed (Red Interuniversitaria Euroamericana de Investigación en Competencias Mediáticas para la Ciudadanía), y del proyecto "Competencia mediática en la sociedad digital para la participación crítica de la ciudadanía. Diagnóstico y planes de acción didácticos" financiado por la Universidad de Cantabria. También ha recibido apoyo del programa de Investigación e Innovación Horizon 2020 de la Unión Europea, bajo el acuerdo Horizon 2020- Seac-20151-710577. 


\section{Referencias}

Acosta-Silva, D. A. (2017). Tras las competencias de los nativos digitales: avances de una metasíntesis. Revista Latinoamericana de Ciencias Sociales, Niñez y Juventud, 15(1), 471-489. https:// doi.org/10.11600/1692715x.1513014062016

Aguaded, I., Caldeiro, M. C., y Rodríguez, J. (2015). ¿Qué nos muestran las pantallas? La mirada crítica adolescente en el marco de las industrias culturales y del pensamiento actual. Alteridad. Revista de Educación, 10(1), 8-20. https://doi. org/10.17163/alt.v10n1.2015.01

Anguita, J. C., Labrador, J. R., Campos, J. D., Casas Anguita, J., Repullo Labrador, J., y Donado Campos, J. (2003). La encuesta como técnica de investigación. Elaboración de cuestionarios y tratamiento estadístico de los datos (I). Atención primaria, 31(8), 527-538.

Benítez, M. E., Manrique, S., y De Mier, V. (2014). Las dimensiones sociales, cognitivas y lingüísticas del proceso de alfabetización en jóvenes y adultos. Revista Interamericana de Educación de Adultos Año, 37(1), 89-106. Recuperado de: https://goo. $\mathrm{gl} / \mathrm{xAyBX5}$

Buckingham, D. (2008). Más allá de la tecnología. Aprendizaje infantil en la era de la cultura digital. Buenos Aires: Ediciones Manantial.

Cabero, J., y Guerra, S. (2011). La alfabetización y formación en medios de comunicación en la formación inicial del profesorado. Educación XX1, 14(1). Recuperado de: https://goo.gl/ TvQgHQ

Cabra, F., y Marciales, G. (2009). Mitos, realidades y preguntas de investigación sobre los nativos digitales: una revisión. Revista Universitas Phychologica, 8(2), 323-338. Recuperado de: https://goo.gl/I12fiI

Caldeiro, M. C., y Aguaded, I. (2015). “Estoy aprendiendo, no me molestes". La competencia mediática como forma de expresión crítica de nativos e inmigrantes digitales. Redes.com (12), 27-45. https://doi.org/10.15213/redes.n12.p26

Carrasco, M. E. E., Sánchez, C., y Carro, A. (2015). Las competencias digitales en estudiantes del posgrado en educación. Revista Lasallista de Investigación, 12, (2), 10-18. Recuperado de: https://goo.gl/VyX3ys

Castellanos, A., Sánchez, C., y Calderero, J. F. (2017). Nuevos modelos tecnopedagógicos. Competencia digital de los alumnos universitarios. Revista Electrónica de Investigación Educativa, 19(1), 1-9. Recuperado de: https://goo.gl/vHHpmM

Comisión Europea (2009). Commission recommendation of 20 August 21011 on media literacy in the digital environment for a more competitive audiovisual and content industry and an inclusive knowledge society. Official Journal of the European Union, L. 227/9, (08/22/2009). Recuperado de: https:// goo.gl/ofZwbz

Comisión Europea (2011). Testing and Refining Criteria to Assess Media Literacy Levels in Europe. Final Report. European Commission. Recuperado de: https://goo.gl/PGGulu

De Casas-Moreno, P., Caldeiro, M. C., y Romero-Rodríguez, L. M. (2018). La televisión como espejo de la realidad del "bullying". Percepciones de los adolescentes sobre el programa "Proyecto Bullying". Aula Abierta, 47(2), 193-202- Recuperado de: https://goo.gl/hdPwWQ

De Oliveira, L. (2016). Focus on digital competence: teaching practice with virtual environment support. Ilha do Desterro, 60(1), 127-140. Recuperado de: https://goo.gl/1BSPTn

Del Valle-Rojas, C., Denegri-Coria, M. \& Chávez-Herting, D. (2012). Media Literacy and Consumption of Media and Advertising in University Students of Pedagogy in Chile. [Alfa- betización audiovisual y consumo de medios y publicidad en universitarios de Pedagogía en Chile]. Comunicar, 38, 183191. https://doi.org/10.3916/C38-2012-03-10

Diego-Mantecón, J. M. (2013). Evaluación de un modelo de creencias transcultural para el aprendizaje de las matemáticas. $G a-$ ceta de la Real Sociedad Matemática Española, 16(3), 561-574.

European Council (2006). Recommendation of the European Parliament and the Council of 18 December 2006 on key competences for lifelong learning. Official Journal of the European Union, 30th December 2006, number 394, pp. 10-18.

Fajardo, I., Villalta, E., y Salmerón, L. (2016). ¿Son realmente tan buenos los nativos digitales?: relación entre las habilidades digitales y la lectura digital. Anales de Psicología, 32(1), 89-97. Recuperado de: https://goo.gl/gjHfb5

Ferrés, J. (2017) La competencia mediática y emocional de los jóvenes. Revista de estudios de juventud, 101, 89-101. Recuperado de: https://goo.gl/31NXeq

Ferrés, J., y Piscitelli, A. (2012). La competencia mediática: propuesta articulada de dimensiones e indicadores Comunicar, 19(38), 75-82. https://doi.org/10.3916/C38-2012-02-08

García-Galera, M. C., \& Valdivia, A. (2014). Prosumidores mediáticos.: Cultura participativa de las audiencias y responsabilidad de los medios. Comunicar, 43, 10-13. http://dx.doi. org/10.3916/C43-2014-a2

García-Ruiz, R., Ramírez, A. \& Rodríguez, M. M. (2014). Media Literacy Education for a New Prosumer Citizenship. [Educación en alfabetización mediática para una nueva ciudadanía prosumidora]. Comunicar, 43, 15-23. https://doi.org/10.3916/ C43-2014-01

Geller, D., Vinokurov, A., y Martin, T. L. (2005). Cross-Cultural Issues in Survey Translation: Translation of Meaning and Meaning of Translation. Paper presented at the Federal Committee on Statistical Methodology conference.

González-Fernández, N., Ramírez-García, A, y Salcines-Talledo, I. (2017). Competencia mediática y necesidades de alfabetización audiovisual de docentes y familias españolas. Educación XX1. Recuperado de: https://goo.gl/M9fFpx

Gozálvez, V., y Aguaded, I. (2012). Educación para la autonomía en sociedades mediáticas. Análisis, (45), 1-14. Recuperado de: https://goo.gl/7dS796

Gozálvez, V., García-Ruiz, R., y Aguaded, I. (2014). La formación en competencias mediáticas: una cuestión de responsabilidad ética en educación superior. Revista Interuniversitaria de Formación del Profesorado, 28(1), 17-28. Recuperado de: $\underline{\text { https:// }}$ goo.gl/pwIYoW

Grijalva. A. A., y Moreno, D. (2016). Competencia mediática en jóvenes universitarios: análisis socieducativo del currículum escolar. Sociología y Tecnociencia, 6(1), 14-25. Recuperado de: https://goo.gl/WzKe1y

Grijalva, A. A., y Urrea, M. L. (2017). Evaluación de la competencia digital en estudiantes de comunicación. El caso de una universidad mexicana. EDMETIC, 6(2), 276-301.Recuperado de: $\underline{\text { https://goo.gl/XCPpBN }}$

Gutiérrez, A. \& Tyner, K. (2012). Media Education, Media Literacy and Digital Competence. [Educación para los medios, alfabetización mediática y competencia digital]. Comunicar, 38, 31-39. https://doi.org/10.3916/C38-2012-02-03

Iglesias, M., y González, C. (2012). Radiografía del consumo de medios de comunicación en estudiantes universitarios. ICONO14, 10(3), 100-115. Recuperado de: https://goo.gl/SJospt

Izquierdo, B., Renés, P. y Gómez Cash, O. (2013). La alfabetización mediática en la universidad a través de talleres multimodales de lectura y escritura académicas. Edmetic, 2(1), 7694. Recuperado de: https://goo.gl/e3wzs3 
Kennedy, G., Dalgarno, B., Gray, K., Judd, T., Waycott, J., Bennett, S. J., ... Churchwood, A. (2007). The Net Generation are not big users of Web 2.0 technologies: preliminary findings. En R. Atkinson, C. McBeath, S. Soong \& C. Cheers (Eds.), Annual Conference of the Australasian Society for Computers in Learning in Tertiary Education (pp. 517-525). Singapore: Nanyang Technology University. Recuperado de: https://goo.gl/HFakVd

Liesa, M., Vázquez, S., y Lloret, J. (2016). Identificación de las fortalezas y debilidades de la competencia digital en el uso de aplicaciones de internet del alumno de primer curso del Grado de Magisterio. Complutense de Educación, 27(2), 845. Recuperado de: https://goo.gl/YwkAfU

López, L. \& Aguaded, M. C. (2015). Teaching Media Literacy in Colleges of Education and Communication. Comunicar, 44, 187-195. https://doi.org/10.3916/C44-2015-20

Ministerio de Educación, Cultura y Deporte (2015). Orden ECD/65/2015, de 21 de enero, por la que se describen las relaciones entre las competencias, los contenidos y los criterios de evaluación de la educación primaria, la educación secundaria obligatoria y el bachillerato. Boletín Oficial del Estado, 29 de enero de 2015, 25, pp. 6986 - 7003. Recuperado de: https:// goo.gl/aN6ohZ

Monreal, I. M., Parejo, J. L., y de la O. M. (2017) Alfabetización mediática y cultura de la participación: retos de la ciudadanía digital en la Sociedad de la Información. EDMETIC, 6(2), 148 167. Recuperado de: https://goo.gl/Kh8bV4

Nachar, N. (2008). The Mann-Whitney U: A test for assessing whether two independent samples come from the same distribution. Tutorials in Quantitative Methods for Psychology, 4(1), 13-20.

Ortega, F., González Ispierto, B. \& Pérez Peláez, M. E. (2015). Audiencias en revolución, usos y consumos de las aplicaciones de los medios de comunicación en tabletas y teléfonos inteligentes. Revista Latina de Comunicación Social, 70, 627-651. https://doi.org/10.4185/RLCS- 2015-1063

Pallant, J., y Manual, S. S. (2010). A step by step guide to data analysis using SPSS. Berkshire UK: McGraw-Hill Education.

Pérez-Rodríguez, A., y Delgado-Ponce, A. (2012) De la competencia digital y audiovisual a la competencia mediática: dimensiones e indicadores. Comunicar, 39, 25-34. https://doi. org/10.3916/C39-2012-02-02

Prensky, M. (2001). Nativos e inmigrantes digitales. Adaptación al castellano del texto original "Digital Natives, Digital Immigrants" Institución Educativa SEK. Recuperado de: http:// goo.gl/8DA7t
Ricoy, M., y Da Silva, M. J. (2016). Dispositivos móveis digitais e competências para a utilização na "sociedade do conhecimento". Convergencia-Revista de Ciencias Sociales, 23(70), 5985. Recuperado de: https://goo.gl/srKLUZ

Rotta, A. L. (2015). Jóvenes y nuevas tecnologías: el futuro de los derechos humanos. Revista de Estudios de Juventud, (107), 7990. Recuperado de: https://goo.gl/zb1bFw

Sánchez-Carrero, J., y Contreras-Pulido, P. (2012). De cara al prosumidor: producción y consumo empoderando a la ciudadanía 3.0. ICONO14, 10(3), 62-84. Recuperado de: https://goo. $\mathrm{gl} / \mathrm{kTwPqv}$

Sarsa, J. (2014). El perfil prosumidor de los estudiantes en la web 2.0. Journal for Educators, Teacher and Trainers, 5(2), 74-87. Recuperado de: https://goo.gl/ECW3n8

Scolari, C. A. (2016). Alfabetismo transmedia: estrategias de aprendizaje informal y competencias mediáticas en la nueva ecología de la comunicación. Telos: Revista de pensamiento sobre Comunicación, Tecnología y Sociedad, 193, 13-23. Recuperado de: https://goo.gl/CWiySB

Sesento, L. (2015). La influencia de los medios de comunicación en los jóvenes. Contribuciones a las Ciencias Sociales, 29. Recuperado de: https://goo.gl/B4y

Soep, E. (2012). The Digital Afterlife of Youth-Made Media: Implications for Media Literacy Education. [Generación y recreación de contenidos digitales por los jóvenes: implicaciones para la alfabetización mediática]. Comunicar, 38, 93-100. https://doi.org/10.3916/C38-2012-02-10

Tyner, K., Gutiérrez Martín, A., \& Torrego González, A. (2015). “Multialfabetización" sin muros en la era de la convergencia. La competencia digital y "la cultura del hacer" como revulsivos para una educación continua. Profesorado. Revista de $\mathrm{Cu}$ rrículum y Formación de Profesorado, 19(2), 41-56. Recuperado de: $\underline{\text { https://goo.gl/cb9H9E }}$

UNESCO (2008). Teacher training curricula for media and information literacy. Report of the International Expert Group Meeting. Paris: International UNESCO. Recuperado de: https://goo. $\mathrm{gl} / 2 \mathrm{tVKEL}$

Valverde, O., Reyes, S. Á., y Espinosa Sarría, E. (2013). Diseño de curso básico para la alfabetización informacional de profesores. Educación Médica Superior, 27(2), 194-202. Recuperado de: https://goo.gl/wB5Czo

Wells, C., y Wollack, J. (2003). An Instructor's Guide to Understanding Test Reliability. University of Wisconsin: Testing \& Evaluation Services. 